\title{
ON A GLOBAL CLASSICAL SOLUTION OF A QUASILINEAR HYPERBOLIC EQUATION
}

\author{
Y. EBIHARA \\ Department of Applied Mathematics \\ Faculty of Science, Fukuoka University \\ Fukuoka 814-01 Japan

\section{D.C. PEREIRA} \\ Instituto de Mathematica-UFRJ \\ CP 68530, CEP 21944 \\ Rio de Janeiro-Bras 11 \\ (Recelved June 16, 1987)
}

ABSTRACT. In this paper we establish the existence and uniqueness of global classical solutions for the equation which arises in the study of the extensional vibrations of thin rod, or torsional vibrations of thin rod.

1. INTRODUCTION.

In this paper we study the existence and uniqueness of global classical solutions of the first initial-boundary value problem for the equation:

$$
u_{t t}-\Delta u-M\left(\int_{\Omega}|\nabla u|^{2} d x\right) \Delta u_{t t}=\mathbf{f}
$$

in $Q=\Omega \times] 0, T\left[\right.$, where $\Omega\left(\partial \Omega\right.$ : the boundary) is a smooth bounded domain in $R^{n}, T$ is a positive number, $\nabla u$ is the gradient of $u, \Delta$ is the Laplace operator and $M(\lambda), \lambda>0$, is a real valued function with $M(\lambda)>\rho>0, \lambda>0$, for some $\rho>0$. We have mathematical interest in solving the equation (1.1) by the following reasons.

F1rst, the equation (1.1) with $M(\lambda) \equiv 1$ arises in the study of the extensional vibrations of thin rods, see Love [1], and 1t was studied by one of the authors in [2] and [3]. Second, the equation (1) with $M(\lambda) \equiv \lambda_{0}, \lambda_{0}=\int_{\delta \Omega} \phi^{2} \mathrm{~d} x$, where $\phi$ is the torsion-function arises in the study of the torsional vibrations of thin rods, see Love [1]. Third, the function $M(\lambda)$ in (1.1) has its motivation in the mathematical description of the vibrations of an elastic stretched string, that is, the equation:

$$
u_{t t}-M\left(\int_{\Omega}|\nabla u|^{2} d x\right) \Delta u=0
$$


what for $M(\lambda)>\rho>0$ was studied by Pohozaev [4], Nishihara [5] and Lions [6]. When $M(\lambda)>0$ was treated by Arosio-Spagnolo [7], Ebihara-Medeiros-Milla Mi randa [8] and Yamada [9].

In this paper, we establish the existence and uniqueness of global classical solutions for the equation (1.1). For that we use the Faedo-Galerkin method and compactness argument with some technical idea.

2. NOTATIONS, ASSUMPTIONS AND MAIN RESULT.

Let $\left(w_{j}\right)_{j \in N}$ be a system of eigen functions of $-\Delta$ which is defined on $H^{2}(\Omega) \cap H_{0}^{1}(\Omega)$. We denote by $V \equiv V(\Omega)$ the set of all finite linear combinations of $\left(w_{j}\right)_{j \in N}$. Putting $(f, g)=\int_{\Omega} f(x) g(x) d x$, we set $(., . .)_{m}=\left((-\Delta)^{m}, \ldots\right), m=1,2, \ldots$, then $(., . .)_{m}$ define an inner product on $v$. We put $v_{m} \equiv v_{m}(\Omega)$ as the closure of $v$ by the topology of norm $|\cdot|_{m}^{2}=(., .)_{m}$. Then we see that

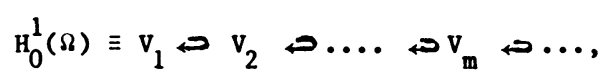

$v_{m} \subset H^{m}(\Omega), m=1,2, \ldots$, and the norm $|\cdot|_{m}$ is equivalent in $v_{m}$ to the standard norm

of $\mathrm{H}^{\mathrm{m}}(\Omega)$. We see that all the above infections "حه" are compact.

Let $T$ be a positive number and $B$ a Banach space with a norm $\|\cdot\|_{B}$. We shall represent by $L^{P}(0, T ; B), 1<P<\infty$, the Banach space of vector-valued functions $\left.\varepsilon_{u}:\right] 0, t\left[+B\right.$ which are measurable in $B$ and $\|u(t)\|_{B} \varepsilon L^{p}(0, T)$ with the norm

$$
\|\mathrm{u}\|_{\mathrm{L}^{\mathrm{P}}(0, \mathrm{~T} ; \mathrm{B})}^{\mathrm{P}} \equiv \int_{0}^{\mathrm{T}}\|\mathrm{u}(\mathrm{t})\|_{B}^{\mathrm{P}} \mathrm{dt}
$$

and by $L^{\infty}(0, T ; B)$ the Banach space of vector-valued functions $\left.u:\right] 0, T[+B$ which are measurable in $B$ and $\|u(t)\|_{B} \in L^{\infty}(0, T)$ with the norm

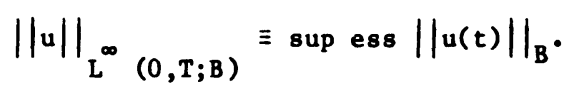

We denote by $C^{j}(0, T ; B)$ the space of all vector-valued functions $u:[0, T] \rightarrow B$, which are $j$-times differentiable in the sense of $B$.

Let $M(\lambda), \lambda>0$ be a real valued function such that:

$$
M(\lambda) \varepsilon C^{1}[0, \infty) \text { and there exist constants } \alpha>0 \text { and } \rho>0 \text { that }
$$

verify $M(\lambda)>\alpha \lambda^{1 / 2}+\rho, \forall \lambda \varepsilon[0, \infty)$, 
(A.2) $\quad\left|M^{\prime}(\lambda)\right| \lambda^{1 / 2}<B(\lambda) M(\lambda)$ where $B(\lambda) \varepsilon c^{0}[0, \infty), B(\lambda)>0, \lambda>0$, then we have the following result:

THEOREM 2.1. Suppose that

$$
\begin{aligned}
& u_{0}, u_{1} \in v_{m},(m>2), \\
& f, f^{\prime} \in C\left(0, T ; v_{m-1}\right)
\end{aligned}
$$

Then there exists a unique function $u:[0, T]+L^{2}(\Omega)$ in the class:

$$
u \varepsilon c^{2}\left(0, T ; v_{m}\right)
$$

that verifies

$$
\begin{aligned}
& u^{\prime \prime}-\Delta u-M\left(|u|_{1}^{2}\right) \Delta u^{\prime \prime}=f \text { in } Q \\
& u(0)=u_{0} \\
& u^{\prime}(0)=u_{1} \\
& \left.u\right|_{\partial \Omega}=0
\end{aligned}
$$

\section{PROOF OF THEOREM 1.}

We divide the proof in four parts:
a) Approximated solutions
b) A priori estimates
c) Passage to the $11 \mathrm{mit}$
d) Uniqueness

a) AP PROXIMATED SOLUTIONS. of $-\Delta$.

Let $\left[w_{1}, \ldots, w_{k}\right]$ be the subspace of $v$, generated by the first $k$ eigenvectors Let

$$
u_{k}(t) \equiv \sum_{j=1}^{k} g_{j k}(t) w_{j} \varepsilon\left[w_{1}, \ldots, w_{k}\right]
$$

be a solution of the system:

$$
\begin{gathered}
\left(u_{k}^{\prime \prime}-\Delta_{u_{k}}-M\left(\left|u_{k}\right|_{1}^{2}\right) \Delta u_{k}^{\prime \prime}, w\right)=(f, w) \text { for a11 w }\left[w_{1}, \ldots, w_{k}\right] \\
u_{k}(0)=u_{0 k} \rightarrow u_{0} \text { strongly in } v_{m} \text { as } k+\infty, \\
u_{k}^{\prime}(0)=u_{1 k}+u_{1} \text { strongly in } v_{m} \text { as } k \rightarrow \infty, \\
\text { where } u_{0 k}=\sum_{j=1}^{k}\left(u_{0}, w_{j}\right) w_{j}, u_{1 k}=\sum_{j=1}^{k}\left(u_{1}, w_{j}\right) w_{j} \cdot
\end{gathered}
$$


Then we see that the solution $u_{k}(t)$ exists on an interval $\left[0, T_{k}\right), 0<T_{k}<T$. A priori estimates will permit us to extend $u_{k}(t)$ to all interval $[0, T]$.

b) A PRIORI ESTIMATES

we have I) Putting $w=u_{k}^{\prime \prime}(t)$ in (3.1),

$$
\left|u_{k}{ }^{\prime \prime}\right|_{0}^{2}+\left(u_{k}, u_{k}^{\prime \prime}\right)_{1}+M\left(\left|u_{k}\right|_{1}^{2}\right)\left|u_{k}^{\prime \prime}\right|_{1}^{2}=\left(f, u_{k}^{\prime \prime}\right) \text {. }
$$

Thus by (A.1),

$$
\begin{aligned}
\frac{\left|u_{k}^{\prime \prime}\right|_{0}^{2}}{M\left(\left|u_{k}\right|_{1}^{2}\right)}+\left|u_{k}^{\prime \prime}\right|_{1}^{2} & <\frac{\left|u_{k}\right|_{1}\left|u_{k}^{\prime \prime}\right|_{1}}{\alpha\left|u_{k}\right|_{1}}+\frac{|f|_{0}\left|u_{k}^{\prime \prime}\right|_{0}}{\rho} \\
& <\frac{1}{\alpha^{2}}+\frac{1}{4}\left|u_{k}^{\prime \prime}\right|_{1}^{2}+\frac{c^{2}|f|_{0}^{2}}{\rho^{2}}+\frac{1}{4}\left|u_{k}^{\prime \prime}\right|_{1}^{2}
\end{aligned}
$$

where $c$ is a positive constant such that $\left|u_{k}^{\prime \prime}\right|_{0}<c\left|u_{k}^{\prime \prime}\right|_{1}$. Then from this and (2.2),

$$
\left|u_{k}^{\prime \prime}\right|_{1}^{2}<2\left(\frac{1}{\alpha^{2}}+\frac{c^{2}|f|_{0}^{2}}{\rho^{2}}\right)<c_{1}
$$

where $c_{1}>0$ is a constant independent of $t$ and $k$.

Thus

$$
\left(u_{k}^{\prime \prime}\right) \text { is bounded in } L^{\infty}\left(0, T_{k} ; V_{1}\right) \text {. }
$$

By Fundamental Theorem of Calculus, we have

and

$$
\left(u_{k}^{\prime}\right) \text { is bounded in } L^{\infty}\left(0, T_{k} ; v_{1}\right)
$$

$$
\left(u_{k}\right) \text { is bounded in } L^{\infty}\left(0, T_{k} ; V_{1}\right) \text {. }
$$

The above estimates permit us to extend $u_{k}(t)$ to all interval $[0, T]$.

$$
\text { II) Making } w=(-\Delta)^{m-1} u_{k}^{\prime} \text {, in (3.1) }
$$

we have:

$$
\begin{aligned}
& \frac{1}{2} \frac{d}{d t}\left[\left|u_{k}^{\prime}\right|_{m-1}^{2}+\left|u_{k}\right|_{m}^{2}+M\left(\left|u_{k}\right|_{1}^{2}\right)\left|u_{k}^{\prime}\right|_{m}^{2}\right] \\
& =M^{\prime}\left(\left|u_{k}\right|_{1}^{2}\right)\left(u_{k}, u_{k}^{\prime}\right)_{1}\left|u_{k}^{\prime}\right|_{m}^{2}+\left(f, u_{k}^{\prime}\right)_{m-1}
\end{aligned}
$$

By (A.2) and (3.6), we have 


$$
\begin{aligned}
& M^{\prime}\left(\left|u_{k}\right|_{1}^{2}\right)\left(u_{k}, u_{k}^{\prime}\right)_{1}\left|u_{k}^{\prime}\right|_{m}^{2}<B\left(\left|u_{k}\right|_{1}^{2}\right) M\left(\left|u_{k}\right|_{1}^{2}\right)\left|u_{k}^{\prime}\right|_{1}\left|u_{k}\right|_{m}^{2} \\
& <\frac{C_{2}}{2} M\left(\left|u_{k}\right|_{1}^{2}\right)\left|u_{k}^{\prime}\right|_{m}^{2} .
\end{aligned}
$$

Then integrating from 0 to $t$ and using (2.1) and (2.2) we obtain:

$$
\begin{aligned}
& \left|u_{k}^{\prime}\right|_{m-1}^{2}+\left|u_{k}\right|_{m}^{2}+M\left(\left|u_{k}\right|_{1}^{2}\right)\left|u_{k}^{\prime}\right|_{m}^{2} \\
& <c_{3}+c_{4} \int_{0}^{t}\left[\left|u_{k}^{\prime}\right|_{m-1}^{2}+M\left(\left|u_{k}\right|_{1}^{2}\right)\left|u_{k}^{\prime}\right|_{m}^{2}\right] d s
\end{aligned}
$$

where $c_{3}=C_{3}\left(u_{0}, u_{1}, T\right)$ and $c_{4}=\max \left\{1, C_{2}\right\}$.

Thus, by Gronwall inequality and (A.1), we have:

$$
\left|u_{k}^{\prime}\right|_{m-1}^{2}+\left|u_{k}\right|_{m}^{2}+\rho\left|u_{k}^{\prime}\right|_{m}^{2}<c_{5}
$$

where $C_{5}>0$ is a constant independent of $t$ and $k$.

Whence,

$$
\begin{aligned}
& \left(u_{k}\right) \text { is bounded in } L^{\infty}\left(0, T ; V_{m}\right) \text {, } \\
& \left(u_{k}^{\prime}\right) \text { is bounded in } L^{\infty}\left(0, T ; V_{m}\right) \text {. }
\end{aligned}
$$

(III) Taking the derivative of (3.1) with respective to $t$, we obtain:

$$
\begin{aligned}
& \left(u_{k}{ }^{(3)}-\Delta u_{k}^{\prime}-M\left(\left|u_{k}\right|_{1}^{2}\right) \Delta u_{k}(3)\right. \\
& \left.-2 M^{\prime}\left(\left|u_{k}\right|_{1}^{2}\right)\left(u_{k}, u_{k}^{\prime}\right), \Delta u_{k}^{\prime \prime}, w\right)-\left(f^{\prime}, w\right) \text { for all w } \varepsilon\left[w_{1}, \ldots, w_{k}\right] .
\end{aligned}
$$

Putting in (3.9) $w=(-\Delta)^{m-1} u_{k}^{\prime \prime}$, we have:

$$
\begin{aligned}
& \frac{1}{2} \frac{d}{d t}\left[\left|u_{k}^{\prime \prime}\right|_{m-1}^{2}+\left|u_{k}^{\prime}\right|_{m}^{2}+M\left(\left|u_{k}\right|_{1}^{2}\right)\left|u_{k}^{\prime \prime}\right|_{m}^{2}\right] \\
& \quad+M^{\prime}\left(\left|u_{k}\right|_{1}^{2}\right)\left(u_{k}, u_{k}^{\prime}\right)_{1}\left|u_{k}^{\prime \prime}\right|_{m}^{2}=\left(f^{\prime}, u_{k}^{\prime \prime}\right)_{m-1} \cdot
\end{aligned}
$$

Thus by (3.2) and (3.6),

$$
\begin{aligned}
& \frac{1}{2} \frac{d}{d t}\left[\left|u_{k}^{\prime \prime}\right|_{m-1}^{2}+\left|u_{k}^{\prime}\right|_{m}^{2}+M\left(\left|u_{k}\right|_{1}^{2}\right)\left|u_{k}^{\prime \prime}\right|_{m}^{2}\right] \\
& <\frac{c_{2}}{2} M\left(\left|u_{k}\right|_{1}^{2}\right)\left|u_{k}^{\prime \prime}\right|_{m}^{2}+\frac{1}{2}\left|f^{\prime}\right|_{m-1}^{2}+\frac{1}{2}\left|u_{k}^{\prime \prime}\right|_{m-1}^{2} \cdot
\end{aligned}
$$

Integrating from 0 to $t$, we have:

$$
\left|u_{k}^{\prime \prime}\right|_{m-1}^{2}+\left|u_{k}^{\prime}\right|_{m}^{2}+M\left(\left|u_{k}\right|_{1}^{2}\right)\left|u_{k}^{\prime \prime}\right|_{m}^{2}
$$




$$
\begin{aligned}
& <\int_{0}^{t}\left|f^{\prime}(s)\right|_{m-1}^{2} d s+c_{4} \int_{0}^{T}\left[\left|u_{k}^{\prime \prime}\right|_{m-1}^{2}+M\left(\left|u_{k}\right|_{1}^{2}\right)\left|u_{k}^{\prime \prime}\right|_{m}^{2}\right] d s \\
& +\left|u_{k}^{\prime \prime}(0)\right|_{m-1}^{2}+\left|u_{1 k}\right|_{m}^{2}+M\left(\left|u_{0 k}\right|_{1}^{2}\right)\left|u_{k}^{\prime \prime}(0)\right|_{m}^{2} \cdot
\end{aligned}
$$

We now estimate $\left|u_{k}^{\prime \prime}(0)\right|_{m}$.

Putting $w=(-\Delta)^{m-1} u_{k}^{\prime \prime}(0)$ in $(3.1)$ and tending $t \rightarrow 0$, we obtain:

$$
\begin{aligned}
& \left|u_{k} "(0)\right|_{m-1}^{2}+\left(u_{k}(0), u_{k}^{\prime \prime}(0)\right)_{m}+M\left(\left|u_{0 k}\right|_{l}^{2}\right)\left|u_{k}^{\prime \prime}(0)\right|_{m}^{2} \\
& =\left(f(0), u_{k}^{\prime \prime}(0)\right)_{m-1} .
\end{aligned}
$$

Thus,

$$
\begin{aligned}
&\left|u_{k}^{\prime \prime}(0)\right|_{m-1}^{2}+M\left(\left|u_{0 k}\right|_{1}^{2}\right)\left|u_{k}^{\prime \prime}(0)\right|_{m}^{2} \leqslant \frac{1}{\gamma}\left|u_{0 k}\right|_{m}^{2}+\gamma\left|u_{k}^{\prime \prime}(0)\right|_{m}^{2} \\
&+\frac{1}{2}|f(0)|_{m-1}^{2}+\frac{1}{2}\left|u_{k}^{\prime \prime}(0)\right|_{m-1}^{2}
\end{aligned}
$$

for $\gamma>0$.

Then, by (A.1), (2.2) and (3.2), we have

$$
\left|u_{k}^{\prime \prime}(0)\right|_{m-1}^{2}+2(\rho-\gamma)\left|u_{k}^{\prime \prime}(0)\right|_{m}^{2} \leqslant c_{6}
$$

where $C_{6}>0$ is a constant independent of $t$ and $k$.

Whence, taking $\gamma$ as $0<\gamma<\rho$, we obtain:

$$
\left|u_{k}^{\prime \prime}(0)\right|_{m}^{2}<c
$$

Thus, by (3.10) and (3.11), we have:

$$
\begin{aligned}
& \left|u_{k}^{\prime \prime}\right|_{m-1}^{2}+\left|u_{k}^{\prime}\right|_{m}^{2}+M\left(\left|u_{k}\right|_{1}^{2}\right)\left|u_{k}^{\prime \prime}\right|_{m}^{2} \\
& <c_{7}+c_{4} \int_{0}^{t}\left[\left|u_{k}^{\prime \prime}\right|_{m-1}^{2}+M\left(\left|u_{k}\right|_{1}^{2}\right)\left|u_{k}^{\prime \prime}\right|_{m}^{2}\right] d s .
\end{aligned}
$$

And by Gronwall lemma and (A.1), we obta1n:

$$
\left|u_{k}^{\prime \prime}\right|_{m-1}^{2}+\left|u_{k}^{\prime}\right|_{m}^{2}+\rho\left|u_{k}^{\prime \prime}\right|_{m}^{2}<c_{8}
$$

where $C_{8}>0$ is a constant independent of $t$ and $k$. 
Whence,

$$
\left(u_{k}^{\prime \prime}\right) \text { is bounded in } L^{\infty}\left(0, T ; V_{m}\right) \text {. }
$$

IV) Putting in (3.9) $w=(-\Delta)^{m-1} u_{k}(3)$,

we obtain:

$$
\begin{aligned}
& \left|u_{k}{ }^{(3)}\right|_{m-1}^{2}+\left(u_{k}^{\prime}, u_{k}(3)\right)_{m}+\left.\left.M\left(\left|u_{k}\right|_{1}^{2}\right)\right|_{k}(3)\right|_{m} ^{2} \\
& +2 M^{\prime}\left(\left|u_{k}\right|_{1}^{2}\right)\left(u_{k}, u_{k}^{\prime}\right)_{1}\left(u_{k}^{\prime \prime}, u_{k}(3)\right)_{m}=\left(f^{\prime}, u_{k}^{(3)}\right)_{m-1} \cdot
\end{aligned}
$$

Thus by $(A .1),(A .2),(3.7),(3.8)$ and $(3.12)$, we have:

$$
\begin{aligned}
& \left|u_{k}(3)\right|_{m-1}^{2}+\rho\left|u_{k}(3)\right|_{m}^{2}<\frac{c_{g}}{\gamma}+\gamma\left|u_{k}(3)\right|_{m}^{2}+\frac{1}{2}\left|f^{\prime}\right|_{m-1}^{2} \\
& +\frac{1}{2}\left|u_{k}^{(3)}\right|_{m-1}^{2}
\end{aligned}
$$

Then

$$
\left|u_{k}^{(3)}\right|_{m-1}^{2}+2(\rho-\gamma)\left|u_{k}^{(3)}\right|_{m}^{2}<c_{10}
$$

where $C_{10}>0$ is a constand independent of $t$ and $k$. Whence we can assert that

$$
\left(u_{k}(3) \text { ) is bounded in } L^{\infty}\left(0, T ; V_{m}\right)\right. \text {, }
$$

c) PASSAGE TO THE LIMIT.

By estimates (3.7), (3.8), (3.12) and (3.13) there is a subsequence of $\left(u_{k}\right)_{k \in N}$ what we denote by $\left(u_{k}\right)_{k \varepsilon N}$ and there exists a function $u$, such that:

$$
\begin{aligned}
& \mathrm{u}_{\mathrm{k}} \stackrel{\star}{\rightarrow} \mathrm{u} \text { weak star in } \mathrm{L}^{\infty}\left(0, \mathrm{~T} ; \mathrm{V}_{\mathrm{m}}\right) \\
& u_{k}^{\prime} \stackrel{\star}{\rightarrow} u^{\prime} \text { weak star in } L^{\infty}\left(0, T ; V_{m}\right) \\
& u_{k}^{\prime \prime} \stackrel{\star}{\rightarrow} u^{\prime \prime} \text { weak star in } L^{\infty}\left(0, T ; V_{m}\right)
\end{aligned}
$$

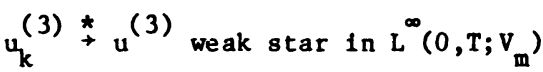

By (3.14) and (3.15), for $m=2$, and since the embedding of $v_{2}$ is compact in $v_{1}$, it follows from Aubin-Lions Theorem [10],

whence

$$
u_{k} \rightarrow u \text { strong } 1 y \text { in } L^{2}\left(0, T ; v_{1}\right)
$$

Now by $(3.16)$,

$$
M\left(\left|u_{k}\right|_{1}^{2}\right) \rightarrow M\left(|u|_{1}^{2}\right) \text { strongly in } L^{2}(0, T)
$$




$$
\Delta u_{k}^{\prime \prime}+\Delta \mathrm{u}^{\prime \prime} \text { weak in } \mathrm{L}^{2}\left(0, \mathrm{~T} ; \mathrm{v}_{\mathrm{m}-2}\right) \text {. }
$$

Thus, by (3.18) and (3.19) we have

$$
M\left(\left|u_{k}\right|_{1}^{2}\right) \Delta u_{k}^{\prime \prime}+M\left(|u|_{1}^{2}\right) \Delta u^{\prime \prime} \text { weak in } L^{2}\left(0, T, v_{m-2}\right)
$$

The above convergences permit us to pass to the $11 \mathrm{mit}$ in the approximate equation (3.1), as $k+\infty$. We then get:

$$
\left(u^{\prime \prime}-\Delta u-M\left(|u|_{1}^{2}\right) \Delta u^{\prime \prime}, w\right)=(f, w)
$$

for each weV in the sense of $L^{2}(0, T)$.

REMARK 1. Since the solution $u$ of $(2.4)$ is in $C^{2}\left(0, T ; V_{m}\right)$, and by Sobolev's theorem

$$
H^{\ell}(\Omega) \subset C^{k}(\bar{\Omega})
$$

with $k<\ell-\frac{n}{2}<k+1, k>0$ integer,

then $u$ satisfies $(2.4)$ in the classic sense if we choose m large enough.

d) UNIQUENESS

Let $u, v$ be solutions of (1.1) In the class of Theorem 1 . Then $w=u-v$ satisfies:

$$
\begin{aligned}
& \omega^{\prime \prime}-\Delta \omega-M\left(|u|_{1}^{2}\right) \Delta \omega^{\prime \prime}-\left[M\left(|v|_{1}^{2}\right)-M\left(|u|_{1}^{2}\right)\right] \Delta v "=0 \\
& \omega(0)=0 \\
& \omega^{\prime}(0)=0
\end{aligned}
$$

Taking of scalar product in $\mathrm{L}^{2}(\Omega)$ of $(3.21)$ by $\omega^{\prime}$, we obtain:

$$
\begin{aligned}
& \frac{1}{2} \frac{d}{d t}\left[\left|\omega^{\prime}\right|_{0}^{2}+|\omega|_{1}^{2}+M\left(|u|_{1}^{2}\right)\left|\omega^{\prime}\right|_{1}^{2}\right]-M^{\prime}\left(\left|u_{1}\right|^{2}\right)\left(u, u^{\prime}\right)_{1}\left|\omega^{\prime}\right|_{1}^{2} \\
& +\left[M\left(|v|_{1}^{2}\right)-M\left(|u|_{1}^{2}\right)\right]\left(v^{\prime \prime}, \omega^{\prime}\right)_{1}=0
\end{aligned}
$$

Now, by (A.1) and (A.2), we have

$$
\begin{aligned}
& \frac{1}{2} \frac{d}{d t}\left[\left.\omega^{\prime}\right|_{0} ^{2}+|\omega|_{1}^{2}+M\left(|u|_{1}^{2}\right)\left|\omega^{\prime}\right|_{1}^{2}\right] \\
& <\frac{C_{1}}{2} M\left(|u|_{1}^{2}\right)\left|\omega^{\prime}\right|_{1}^{2}+M^{\prime}(\xi)\left(|u|_{1}+|v|_{1}\right)|\omega|_{1}\left|v^{\prime \prime}\right|_{1}\left|\omega^{\prime}\right|_{1},
\end{aligned}
$$

where $\xi=(1-\theta)|u|_{1}^{2}+\theta|v|_{1}^{2}, 0<\theta<1$. 
Then,

$$
\frac{1}{2} \frac{d}{d t}\left[\left|\omega^{\prime}\right|_{0}^{2}+|\omega|_{1}^{2}+M\left(|u|_{1}^{2}\right)\left|\omega^{\prime}\right|_{1}^{2}\right]<\frac{C}{2}\left(|\omega|_{1}^{2}+\left|\omega^{\prime}\right|_{1}^{2}\right),
$$

whence by (A.1),

$$
\left|\omega^{\prime}\right|_{0}^{2}+|\omega|_{1}^{2}+\rho\left|\omega^{\prime}\right|_{1}^{2}<c \int_{0}^{t}\left(|\omega|_{1}^{2}+\left|\omega^{\prime}\right|_{1}^{2}\right) d s .
$$

Thus, we have

$$
\omega \equiv 0 \text { in }[0, T]
$$

REMARK 2. In the forthcoming work we will try to study the equation (1.1) when $M(\lambda)$ has zero points, that 1s, degenerate case.

\section{REFERENCES}

1. LOVE, A.H. A treatise on the mathematical theory of elasticity. Dover New York 1944.

2. PEREIRA, D.C. and IZAGUIRRE, R.M. Sobre uma equacao de vibracoes nao 1ineares. Proceedings of 230 seminario Brasileiro de Analise, Campinas, Sao Paulo (1986), 155-158.

3. PEREIRA, D.C. Problema misto para uma equacao de vibracoes nao lineares. To appear.

4. POHOZAEV, S.I. On a class of quasilinear hyperbolic equations. Mat USSR Sbornik 25,1 (1975), 145-148.

5. NISHIHARA, K. On a global solution of some quasilinear hyprbolic equation. Tokyo Journal of Math. I (1984).

6. LIONS, J.L. On some questions in boundary value problems of mathematical physics. IM-UFRJ (1978).

7. AROSIO, A. and SPAGNOLLO, S. Global solutions of the Cauchy Problem for a nonlinear hyperbolic equation. Universita de Pisa, Dipartamento di Mathematica, Roma (1982).

8. EBIHARA, Y., MEDEIROS, L. A. and MILLA MIRANDA, M. Local solutions for a nonlinear degenerate hyperbolic equation. Nonlinear Analysis, Theory Methods and Applications, 10 (1986), 27-40.

9. YAMADA, Y. Some nonlinear degenerate wave equation. To appear in Nonlinear Analysis, Theory, Methods and Applications.

10. LIONS, J.L. Quelques methodes de resolution des problemes aux limites non lineaires. Dunod, Paris, (1969). 


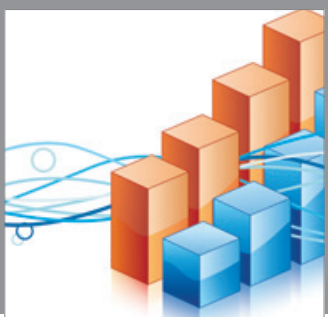

Advances in

Operations Research

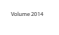

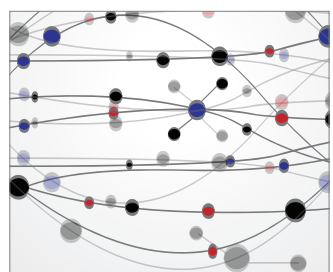

\section{The Scientific} World Journal
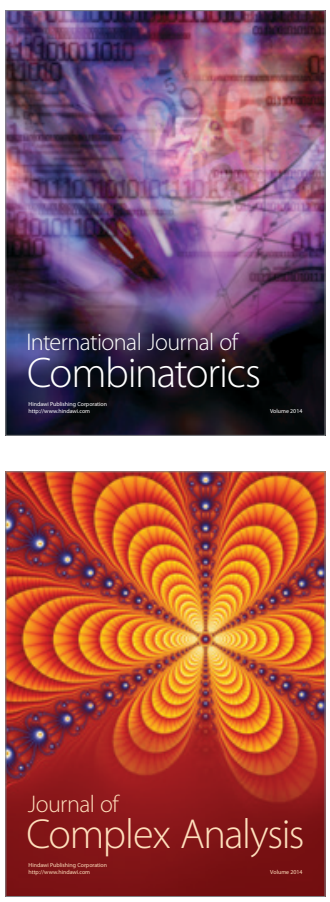

International Journal of

Mathematics and

Mathematical

Sciences
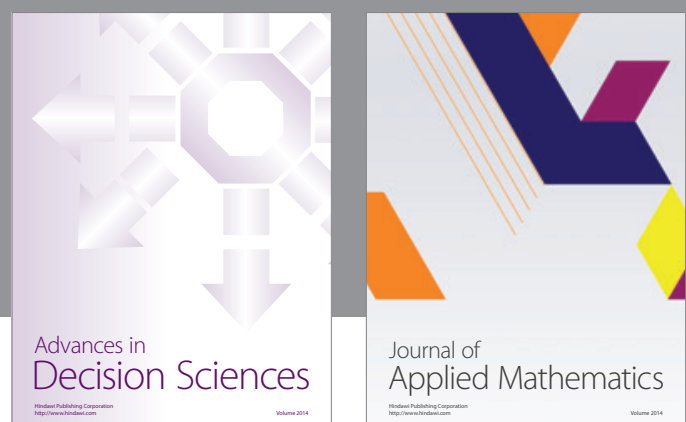

Journal of

Applied Mathematics
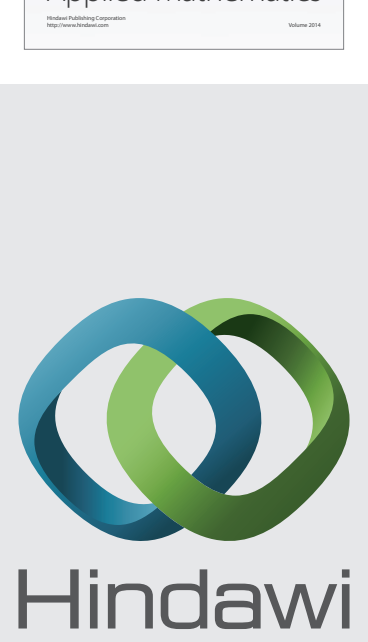

Submit your manuscripts at http://www.hindawi.com
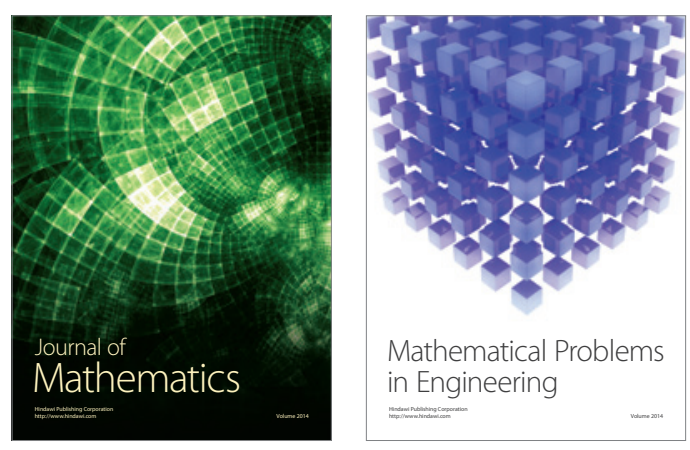

Mathematical Problems in Engineering
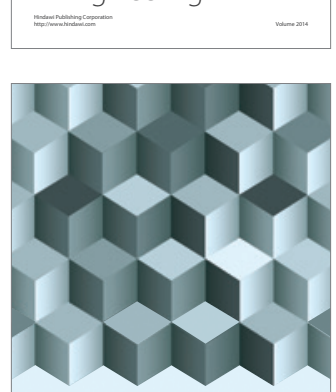

Journal of

Function Spaces
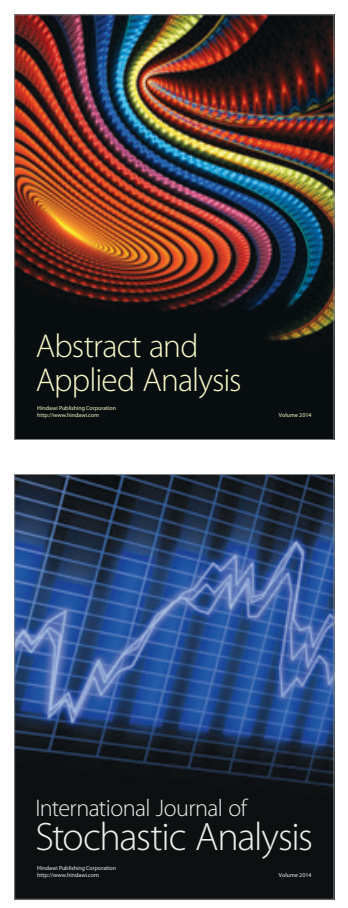

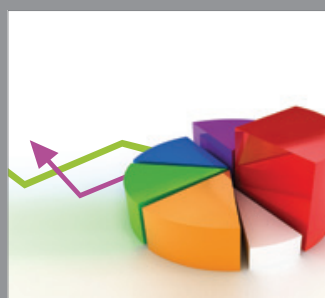

ournal of

Probability and Statistics

Promensencen
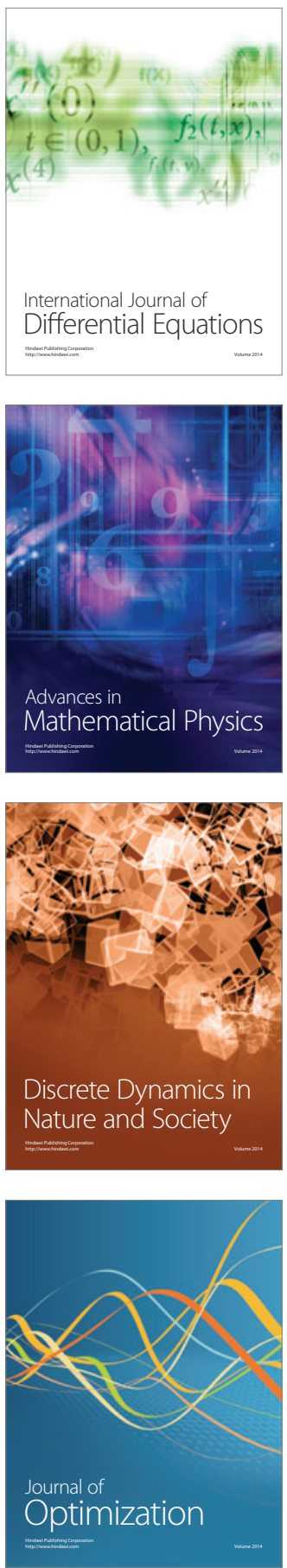